\title{
Cannabis and the developing brain challenge risk perception
}

\author{
Yasmin L. Hurd ${ }^{1,2}$ \\ 'Department of Psychiatry and Department of Neuroscience, Icahn School of Medicine, New York, New York, USA. ${ }^{2}$ Addiction Institute of Mount Sinai, Behavioral Health System, Mount Sinai, New York, New York, USA.
}

$\mathbf{T}_{\mathrm{t}}$ he past decade has seen a reduction in society's perception of cannabis being of any risk or harm. However, we are in a transformational time in which cannabis' health implications related to neurodevelopment, particularly for vulnerable populations, must challenge such perceptions of harmlessness. The combination of multiple interrelated factors - societal, political, and business - have created the "perfect cannabis storm" of unintended neurodevelopmental consequences with an unmatched exposure to cannabis compared with exposure levels never experienced before in human history. Increased exposure is driven by the drug's increased availability across all levels of society. This is due to the legalization of cannabis in most states, coupled with the recent explosion of highly modified cannabis strains and extracted pure $\Delta 9$-tetrahydrocanabinol (THC), the prominent psychoactive cannabinoid from the plant. This explosion has led to diverse products consumed by numerous routes and with extremely high THC potencies. Thus, cannabis today is dramatically different from cannabis even ten years ago, with exponentially increased THC concentrations (1). At the same time, levels of other cannabinoids such as cannabidiol (CBD) that appear to have some protective properties are lower in the plant, leading to THC/CBD ratios of 104 in 2017 versus 23 in 2008 (1). While some turn to dispensaries for "safer" cannabinoids, these products can contain far greater THC content $(70 \%)$ than the cannabis plant ( $17 \%)$. The wide exposure to potent cannabis and cannabinoid extracts across the population particularly heightens the health risk of vulnerable groups associated with sensitive windows of brain development, namely pregnant women, children, adolescents, and emerg- ing adults. Each group carries certain risks relevant to mental health outcomes based on specific features of their stage of brain ontogeny, but cannabis exposure can also enhance vulnerability for all, extending into later stages in life (Figure 1).

\section{Prenatal development, the first cannabis hit}

The aggressive and broad educational programs regarding tobacco and alcohol use during pregnancy have not yet been adapted for cannabis. Instead, pregnant women are even targeted in advertisements and social media about the benefits of so-called medical cannabis to relieve nausea, vomiting, and weight loss associated with morning sickness, as well as to alleviate anxiety and pain. Today, cannabis use by pregnant women in the United States has increased significantly, with a prevalence of more than $7 \%$ overall and $12.1 \%$ during the first trimester (2). Shockingly, approximately $16.2 \%$ of pregnant women aged 18 to 44 years use cannabis nearly every day, with greatest use among those who are young, unmarried, and/or experiencing socioeconomic burdens (3). These numbers likely underestimate the real situation, since many studies are based on self-reports.

Potent, biologically active cannabinoids such as THC readily cross the placental barrier with the potential to impact the fetus. Though not equivocal, multiple reports document that cannabis consumption throughout pregnancy increases the risk of intrauterine growth retardation, low birth weight, and prematurity, particularly with heavy/frequent cannabis use. These reported consequences are not surprising, since the endogenous cannabinoid (also known as endocannabinoid, eCB) system - consisting of cannabinoid

Conflict of interest: The author has declared that no conflict of interest exists.

receptors to which THC binds as well as eCB ligands and the enzymes that regulate their synthesis and degradation - is involved in a broad spectrum of developmental processes at every stage of pregnancy, from implantation to parturition (4). eCB signaling tightly regulates stem cell proliferation, neuron and glial migration, cell differentiation, axon-dendrite polarization, and synaptogenesis, which together orchestrate the hardwiring of the brain (5). As such, eCB perturbation with cannabis exposure could adversely affect neurodevelopment. THC is, however, not normally considered teratogenic, since cannabis exposure during pregnancy does not cause marked congenital defects, e.g., mental retardation and developmental disability, as with fetal alcohol syndrome. Nevertheless, evidence suggests that in utero cannabis exposure during the periconceptional period, approximately the first to fourth weeks of gestation, increases the risk of anencephaly (6). These findings are consistent with preclinical evidence that THC modulates the Sonic hedgehog pathway, which plays a critical role in fetal development, leading to brain dysmorphology (7). Importantly, many women do not know they are pregnant during the periconceptional period, and eCB signaling perturbations have been associated with early pregnancy loss (4).

The risks of in utero cannabis exposure must also be considered in relation to potential long-term and enduring consequences. Pioneering longitudinal studies (e.g., Maternal Health Practices and Child Development Project, Ottawa Prenatal Prospective Study, and Generation R) report neurobehavioral problems related to prenatal cannabis exposure $(8,9)$. Although some noted differences exist between the studies (due to a variety of factors, e.g., cannabis strains, THC concentrations, frequency and duration of use, genetics, and other environmental conditions), all highlight negative long-term outcomes. Importantly, 
Fetal

Infancy

Childhood

Adolescence

Young adults

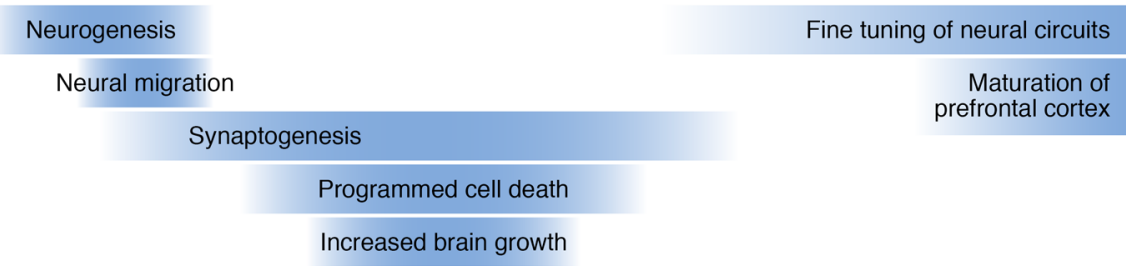

Synaptic pruning

Myelination
Long-term impact on adolescent and adult behavior

\begin{tabular}{|c|c|c|}
\hline \multicolumn{3}{|c|}{ Myelination } \\
\hline $\begin{array}{l}\text { Low birth weight } \\
\text { Altered motor and social- } \\
\text { emotional behaviors } \\
\text { Altered sleep patterns }\end{array}$ & $\begin{array}{l}\text { Altered cognitive, } \\
\text { social, and motor } \\
\text { behaviors }\end{array}$ & $\begin{array}{c}\text { Paranoia } \\
\text { Psychosis } \\
\text { Anxiety } \\
\text { Amotivational syndrome } \\
\text { Short-term memory impairment } \\
\text { Motor coordination impairment } \\
\text { Cannabis use disorder }\end{array}$ \\
\hline
\end{tabular}

\section{Synaptic pruning}

these investigations are complemented by animal studies that causally demonstrate protracted effects of prenatal THC on behaviors during juvenile and adult periods $(5,10)$. Animal models also replicate gene expression alterations detected in the brains of human cannabis-exposed fetuses (11) and provide evidence of cortical rewiring as well as molecular disturbances well into adulthood $(5,10)$.

\section{Infancy and childhood, a} growing developmental window of cannabis exposure

Reduced risk perception has also coincided with increased cannabis use in adults with children at home, and secondhand inhalation is a significant source of cannabis exposure in infancy and childhood (12). Even women who abstain from use while pregnant often restart after pregnancy, and THC and other cannabinoids are transferred to breastmilk during lactation, with potential to impact neurodevelopmental processes. Infancy is characterized by rapid and dynamic brain growth (achieving $~ 80 \%$ of the adult mass) as the size of existing cells increases and trillions of neural connections (i.e., synapses) are formed. This is followed subsequently after age two by a period of pruning and remodeling to improve the efficiency of brain circuits. A fundamental property of $\mathrm{eCB}$ function is at the synapse, where its key role in synaptogenesis during fetal development subsequently switches to tightly modulating synaptic function (5). The health impact of cannabis exposure during early life and childhood remains insufficiently examined in clinical studies. However, animal models demonstrate that THC exposure during lactation leads to sociobehavioral disturbances in adolescence and adulthood and perturbs synaptic plasticity by altering the trajectory of neuronal development within the prefrontal cortex (PFC) $(5,10)$, a brain region highly implicated in several psychiatric illnesses.

\section{Teenagers and emerging adults, a different cannabis era}

The normal nature of adolescence is one of experimentation and risk-taking. However, the current perfect cannabis storm puts adolescents in far greater danger than previous generations, especially given concentrated and toxic administration routes embraced today such as vaping (with cheap black-market devices) and dabbing (providing up to $90 \%$ THC). Although the majority of brain development occurs before age five, the equally critical remaining $10 \%$ of brain development constituting the final organizational phase of maturation extends well into the mid-twenties. This period involves fine-tuning of neural pathways through pruning (elimination of synapses) and stabilization of remaining synapses. The PFC, associated with higher cognitive function and emotional regulation, is the final region to gain full maturity, and the eCB system is an important mediator of proper PFC maturation (13). It is well documented that cannabis use during ado-
Figure 1. Schematic overview of human brain development regulated by the eCB system. Exposure to cannabis at different growth stages from pregnancy to young adulthood (black bar) affects neurodevelopmental processes (blue bars) to impact physiology and behavior (pink boxes). lescence, particularly early and frequent use, is strongly associated with negative long-term outcomes in psychiatric vulnerability and addiction risk $(14,15)$, behavioral outcomes recapitulated in animal models (10). It is also now clear that high-potency cannabis strongly predicts the odds of psychotic disorder (15), substantially altering the life courses of individuals as they head into adulthood (Figure 1).

\section{Conclusions: moving the dial}

The reduced risk perception of cannabis has profoundly affected vulnerable groups. Irrespective of beliefs that adult use is acceptable, as with other recreational drugs such as tobacco and alcohol, and should not be criminalized, the suggestion that cannabis is harmless is invalid, and we must address this misinformation head on. Cannabis today is a highly potent drug, and dose matters. Solutions to the current problems of prenatal, childhood, and early adult cannabis exposure must integrate science in education and government policies to modify perceptions.

Education. Information regarding the integral nature of the eCB system in modulating all stages of neurodevelopment, as well as the risk to that system posed by cannabis exposure, must be strongly incorporated into messages to pregnant women, parents with children at home, and teens and young adults. While potential benefits of medical cannabis have contributed to the reduction in risk perception, confusion exists in the public and for cli- 
nicians regarding terminology. The terms medical marijuana, medical cannabis, and cannabidiol are often used interchangeably although they do not refer to the same thing. Consistent terminologies and definitions must be adopted across all areas (commercial, government, and medical).

Policy. Though influencing the course of cannabis policies will be challenging, it is not too late. Current laws have not deeply considered the health impact for vulnerable groups, only setting a generic ban on purchasing products for those under 21 years old. This ban does little to reduce exposure, especially to high-potency cannabis. Federal and state regulations are still evolving, and nonjudgmental science-based programs are needed. While many states include some efforts to support research from tax revenues associated with cannabis legalization, these efforts have been minimal or unstructured. The inclusion of comprehensive research in all cannabis policy initiatives and medical cannabis/marijuana programs is essential to fully identify those at most risk and help mitigate health outcomes.

Science. Definitive answers regarding the long-term developmental consequences of current cannabis products will not come rapidly from human studies, emphasizing the need for more translational animal studies considering factors mentioned in this Viewpoint, e.g., relevant THC concentrations and evaluation of full-spectrum cannabis. Nevertheless, recently initiated longitudinal projects such as the Adolescent Brain Cognitive Development (ABCD) and HEALthy Brain and Child
Development (HBCD) studies tracking in utero, childhood, and adolescent drug exposures will provide valuable neuroimaging and behavioral health insights. Additional biological measures are also needed in such projects to help identify noninvasive biomarkers potentially predictive of long-term outcomes.

Overall, an integrated science-based strategy can provide a strong foundation for protecting vulnerable groups while affording the advancement of true medical cannabinoid strategies that together improve human health.

\section{Acknowledgments}

YLH is supported by grant DA030359 from $\mathrm{NIH} /$ National Institute on Drug Abuse.

Address correspondence to: Yasmin L. Hurd, Icahn School of Medicine at Mount Sinai, 1 Gustave Levy Place, Box 1639, New York, New York10029, USA. Phone:212.824.9313; Email:yasmin.hurd@mssm.edu.

1. Chandra S, Radwan MM, Majumdar CG, Church JC, Freeman TP, ElSohly MA. New trends in cannabis potency in USA and Europe during the last decade (2008-2017). Eur Arch Psychiatry Clin Neurosci. 2019;269(1):5-15.

2. Volkow ND, Han B, Compton WM, McCanceKatz EF. Self-reported medical and nonmedical cannabis use among pregnant women in the United States. JAMA. 2019;322(2):167-169.

3. Ko JY, Farr SL, Tong VT, Creanga AA, Callaghan WM. Prevalence and patterns of marijuana use among pregnant and nonpregnant women of reproductive age. Am JObstet Gynecol. 2015;213(2):201.e1-201.e10.

4. Cecconi S, Rapino C, Di Nisio V, Rossi G, Maccarrone $\mathrm{M}$. The (endo)cannabinoid signaling in female reproduction: What are the latest advances? Prog Lipid Res. 2020;77:101019.

5. Maccarrone M, Guzmán M, Mackie K, Doherty P, Harkany T. Programming of neural cells by (endo)cannabinoids: from physiological rules to emerging therapies. Nat Rev Neurosci. 2014;15(12):786-801.

6. van Gelder MM, Donders AR, Devine O, Roeleveld N, Reefhuis J, National Birth Defects Prevention Study. Using bayesian models to assess the effects of under-reporting of cannabis use on the association with birth defects, national birth defects prevention study, 1997-2005. Paediatr Perinat Epidemiol. 2014;28(5):424-433.

7. Fish EW, et al. Cannabinoids exacerbate alcohol teratogenesis by a CB1-Hedgehog interaction. Sci Rep. 2019;9(1):16057.

8. Huizink AC. Prenatal cannabis exposure and infant outcomes: overview of studies. Prog Neuropsychopharmacol Biol Psychiatry. 2014;52:45-52.

9. Goldschmidt L, Richardson GA, Larkby C, Day NL. Early marijuana initiation: the link between prenatal marijuana exposure, early childhood behavior, and negative adult roles. Neurotoxicol Teratol. 2016;58:40-45.

10. Hurd YL, Manzoni OJ, Pletnikov MV, Lee FS, Bhattacharyya S, Melis M. Cannabis and the developing brain: insights into its long-lasting effects. J Neurosci. 2019;39(42):8250-8258.

11. DiNieri JA, et al. Maternal cannabis use alters ventral striatal dopamine $\mathrm{D} 2$ gene regulation in the offspring. Biol Psychiatry. 2011;70(8):763-769.

12. Goodwin RD, et al. Trends in cannabis and cigarette use among parents with children at home: 2002 to 2015 . Pediatrics. 2018;141(6):e20173506.

13. Dow-Edwards D, Silva L. Endocannabinoids in brain plasticity: cortical maturation, HPA axis function and behavior. Brain Res. 2017;1654(Pt B):157-164.

14. Silins E, et al. Young adult sequelae of adolescent cannabis use: an integrative analysis. Lancet Psychiatry. 2014;1(4):286-293.

15. Di Forti M, et al. The contribution of cannabis use to variation in the incidence of psychotic disorder across Europe (EU-GEI): a multicentre case-control study. Lancet Psychiatry. 2019;6(5):427-436. 